\title{
Calibration of hydrological model parameters for ungauged catchments
}

\author{
A. Bárdossy \\ IWS, University of Stuttgart, Pfaffenwaldring 61, 70550 Stuttgart, Germany \\ Received: 24 April 2006 - Published in Hydrol. Earth Syst. Sci. Discuss.: 21 June 2006 \\ Revised: 30 November 2006 - Accepted: 6 December 2006 - Published: 17 January 2007
}

\begin{abstract}
The parameters of hydrological models for catchments with few or no discharge records can be estimated using regional information. One can assume that catchments with similar characteristics show a similar hydrological behaviour and thus can be modeled using similar model parameters. Therefore a regionalisation of the hydrological model parameters on the basis of catchment characteristics is plausible. However, due to the non-uniqueness of the rainfallrunoff model parameters (equifinality), a workflow of regional parameter estimation by model calibration and a subsequent fit of a regional function is not appropriate. In this paper a different approach for the transfer of entire parameter sets from one catchment to another is discussed. Parameter sets are considered as tranferable if the corresponding model performance (defined as the Nash-Sutclife efficiency) on the donor catchment is good and the regional statistics: means and variances of annual discharges estimated from catchment properties and annual climate statistics for the recipient catchment are well reproduced by the model. The methodology is applied to a set of 16 catchments in the German part of the Rhine catchments. Results show that the parameters transfered according to the above criteria perform well on the target catchments.
\end{abstract}

\section{Introduction}

Hydrological modelling of water balances or extremes (floods and droughts) is important for planning and water management. Unfortunately the small number (or even the lack) of observations of key variables that influence hydrological processes limits the applicability of rainfall-runoff models; discharge is only measured at a few locations, precipitation measurements are taken at some selected points.

Correspondence to: A. Bárdossy

(bardossy@iws.uni-stuttgart.de)
Thus modelling is an important tool to estimate the elements of the water cycle in areas of interest. In principle, if the models are based on the basic principles of physics (mass and energy conservation), the estimation of model parameters should be a straightforward task. Unfortunately, the extreme heterogeneity of the influencing parameters, such as soil properties or the unresolved spatial and temporal variability of meteorological variables (mainly rainfall), limits the applicability of physically-based models to mainly process studies on small well observed experimental catchments. On meso-scale catchments with observed discharge series, conceptual or partly conceptual models can be used well if they are calibrated on observed events. These types of models are often used for flood forecasting or design purposes. The application of these models on ungauged catchments is very limited as the model parameters are estimated using calibration. The model parameters could be transferred using regression based regionalisation methods (Abdulla and Lettenmaier, 1997; Kokkonen et al., 2003). However the transfer of parameters is difficult as:

- optimal parameter sets depend on the models and the objective functions used to measure their performance (Gupta et al., 1998; Madsen, 2003)

- parameters are themselves uncertain (Kuczera and Mroczkowski, 1998)

- parameters are not unique - a diverse set of possible parameter values can lead to similar model performances (equifinality) (Beven and Freer, 2001).

As model calibration can lead to non-unique sets of parameters, it is difficult to associate the parameters estimated through calibration with the characteristics of the catchment and to transfer them to ungauged locations. In Hundecha and Bárdossy (2004), model parameters were regionalized through simultaneous calibration of the same hydrological

Published by Copernicus GmbH on behalf of the European Geosciences Union. 


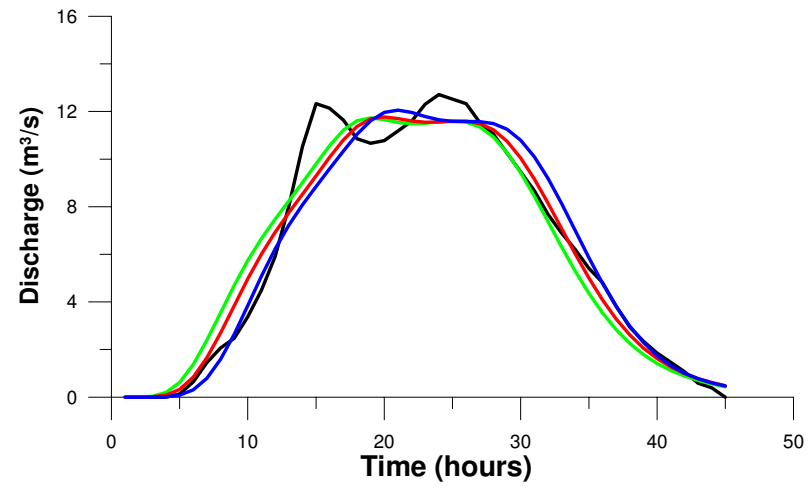

Fig. 1. Observed (solid black) and modelled (red, greed and blue lines) direct discharges for the Kocher at Abtsgmnd, May 1999.

model on different catchments. This procedure however assumes parameters whose dependence can be described using an a priori defined function. Further, only one set of parameters is obtained for the ungauged catchment. The regression approach was strongly criticized in McIntyre et al. (2005). They suggest to investigate the catchment descriptor - model parameter relationship to produce a joint distribution function of the model parameters. The approach requires a large number of observed catchments. Heuvelmans et al. (2006) investigate the use of neural nets for regionalisations. Parajka et al. (2005) use kriging and a similarity based approach to transfer model parameters from one catchment to another.

It would be desirable if - for the triple: a catchment, a model and a parameter set - one could assign a quality metric to help decide how well the model performs on the given catchment by using the selected parameter set. This task however is not realistic.

The purpose of this paper is to develop and to test a simple methodology to transfer hydrological model parameter sets to other catchments as sets, entities or vectors. The idea of using parameter vectors instead of individual parameters is explained with a simple example in the second section of this paper. The properties of those parameter vectors leading to good model performances are investigated in the third section. In the fourth section, the transfer methodology is outlined and applied to 16 selected sub-catchments of the German part of the Rhine catchment. The paper ends with a summary and conclusions.

\section{Interdependence of model parameters}

Hydrological models describe the natural processes of the water cycle. Due to the large complexity of the corresponding natural phenomena, these models contain substantial simplifications. They consist of basic equations, often loosely based on physical premises, whose parameters are specific for the selected catchment and problem under study. For partly or fully conceptual models, some parameters can- not be considered as physically measured (or measurable) quantities and thus have to be estimated on the basis of the available data and information. Due to the fact that in the range of possible (or already observed) input data, different model parameters lead to a similar performance, the identification of a unique dataset is practically impossible (Beven and Freer, 2001). However those model parameter sets which lead to a good model performance might have interesting internal structures. This fact is illustrated with an extremely simple two parameter unit hydrograph model. For a selected flood event in May 1999 on the River Kocher at Abstgmund, the hydrograph of the direct runoff was modelled using a Nash cascade (Nash, 1960) as a unit hydrograph model. Precipitation and discharge were both observed with a $1 \mathrm{~h}$ temporal resolution. The instantaneous unit hydrograph (impulse response function) of the Nash cascade is:

$u(t, k, n)=\frac{1}{k \Gamma(n)}\left(\frac{t}{k}\right)^{n-1} e^{-\frac{t}{k}}$

The Nash cascade is described by two parameters $n$ (number of reservoirs) and $k$ (storage constant). $\Gamma$ is the gamma function and $t$ stands for time. A large number of different parameter combinations were generated, and the performance of the model was estimated using the Nash-Sutcliffe efficiency (NS) (Nash and Sutcliffe, 1970). Figure 1 shows the observed and some of the fitted hydrographs. The hydrographs calculated correspond to different parameter vectors $(n, k)$, but all have nearly the same performance (NS-value). A large number of independent pairs $(n, k)$ were randomly generated without thought of fitting the observed hydrograph and the corresponding NS values were calculated. Figures 2 and 3, respectively, show the model performance for a large number of possible values for the first parameter $(n)$ and for the second parameter $(k)$. These two figures show that there is a large range of values for both parameters, such that for each parameter $k$ (or $n$ ) one can select a second parameter $n$ (or $k$ ) and the model's performance will still be close to optimal. This figure indicates a high uncertainty for both of the parameters. They can be taken from a wide interval of possible values which might lead to good model performance. However, if one investigates the set of parameters as pairs, one obtains a well structured set. Figure 4 shows the pairs of parameters which perform better than $95 \%$ of the NS recorded for the best set. The pairs with good performance all lie along a (hyperbolic) curve indicating that the uncertainty is mainly due to a compensation - for a large range of parameters $k$ one can find a more or less unique parameter $n$ such that the model performs well, the product $n k$ being the collective lag of the cascade. In the case of more complicated models one can assume similar behaviour, however the identification of higher dimensional hyper surfaces of parameters whose points lead to nearly equal performance is much more complicated.

The (usually unknown) non-linear relationships between the parameters and make their transfer to ungauged 


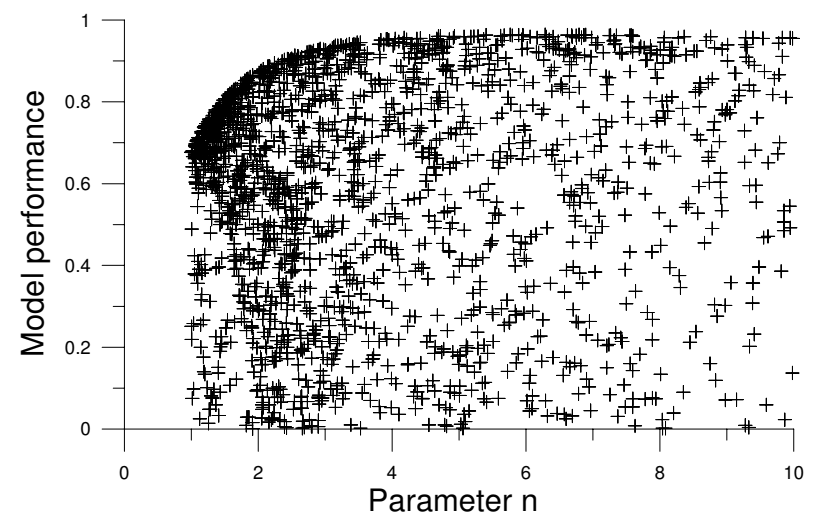

Fig. 2. Model performance (Nash-Sutcliffe) for random parameter sets - as a function of the parameter $n$.

catchments extremely difficult. Imagine one would have three catchments with "good" model parameters around the same line. If a simple optimization of the parameters was carried out the optima for the first two catchments would correspond to points A and B on Fig. 4. If one would interpolate between these points using a linear scheme to find parameters for the third catchment, point $\mathrm{C}$ would be obtained. Despite both model parameter vectors performing well, the interpolated $\mathrm{C}$ is far from the line and would lead to a bad model performance. This simple example shows that the transfer of model parameters of more complex models to ungauged catchments is an extremely difficult task, and that model parameters cannot be treated as independent individual values but instead as complementary parameter vectors.

\section{Transfer of hydrological model parameters}

In this section, the transfer of model parameters of a simple conceptual rainfall-runoff model is considered. A distributed hydrological model based on the HBV (Bergström, 1995) concept was used to explain the methodology. The model consists of different elements describing the relevant hydrological processes. The spatially distributed processes calculated for each zone are:

- snow accumulation and melt, modeled by a degree-day method;

- the proportion of rain or snowmelt that produces runoff as a function of the soil moisture deficit;

- evapotranspiration calculated on the basis of the long term monthly mean values of potential evapotranspiration. This value is adjusted on a daily basis for temperature anomalies using a monthly factor.

The runoff response routine calculates the transformation of the excess water into discharge at the outlet of the subcatchment according to the soil moisture situation. The routine

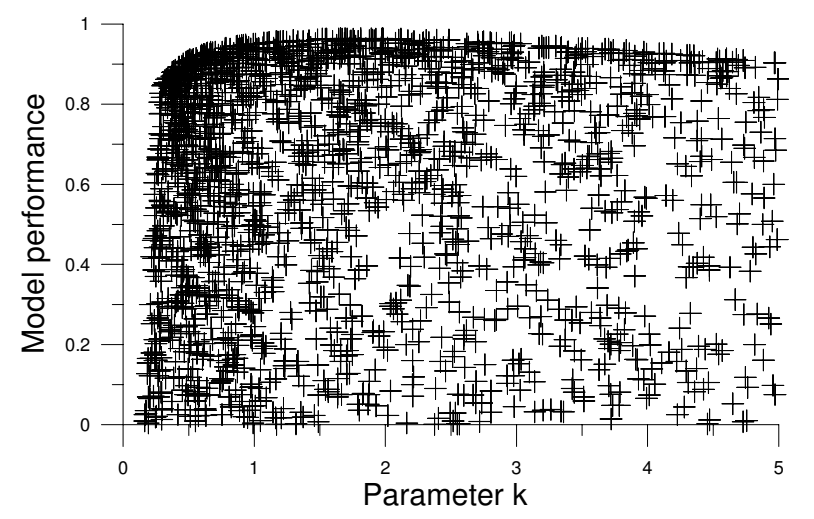

Fig. 3. Model performance (Nash-Sutcliffe) for random parameter sets - as a function of the parameter $k$, fitted after $n$ was randomly selected.

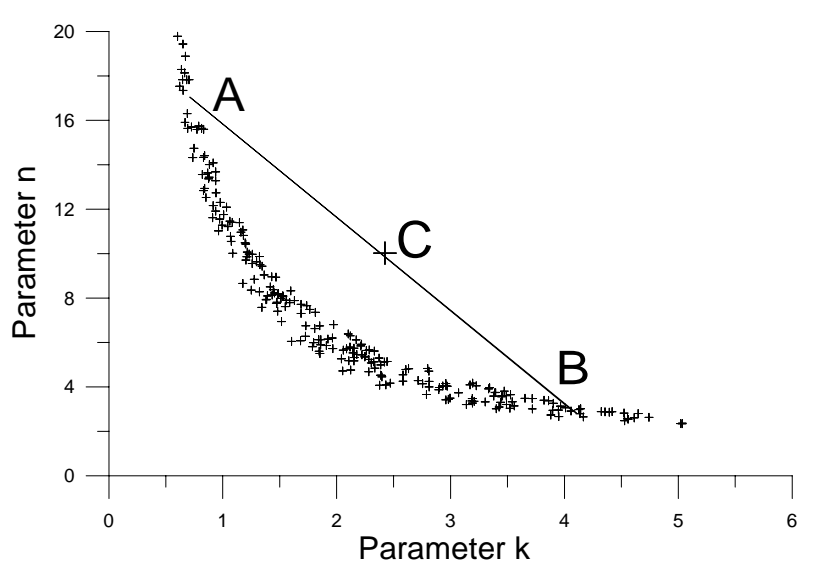

Fig. 4. Model parameters k and n with good performance (NS ¿ $95 \%$ of the maximum NS).

consists of one upper reservoir with two lateral and a vertical outlet and a lower, linear reservoir. The reservoirs are linear and each outlet has a different recession constant. For further details, refer to Hundecha and Bárdossy (2004).

This model was applied to selected meso-scale subcatchments of the German part of the Rhine catchment. The selected catchment sizes vary between 500 and $2500 \mathrm{~km}^{2}$. Daily discharge data from 100 gauging stations, as well as daily temperature data from 150 stations and precipitation data from 601 stations within and around the study area were obtained for the period 1985-2000. Meteorological input for the hydrological model was interpolated from observations with External Drift Kriging (Ahmed and de Marsily, 1987) using topographical elevation considered as external drift. Model parameters related to soil and evapotranspiration were estimated directly for each subcatchment.

A set of 16 subcatchments representing different land use and topographical conditions was selected as a basis for finding transferable parameter sets. Figure 5 shows the locations 


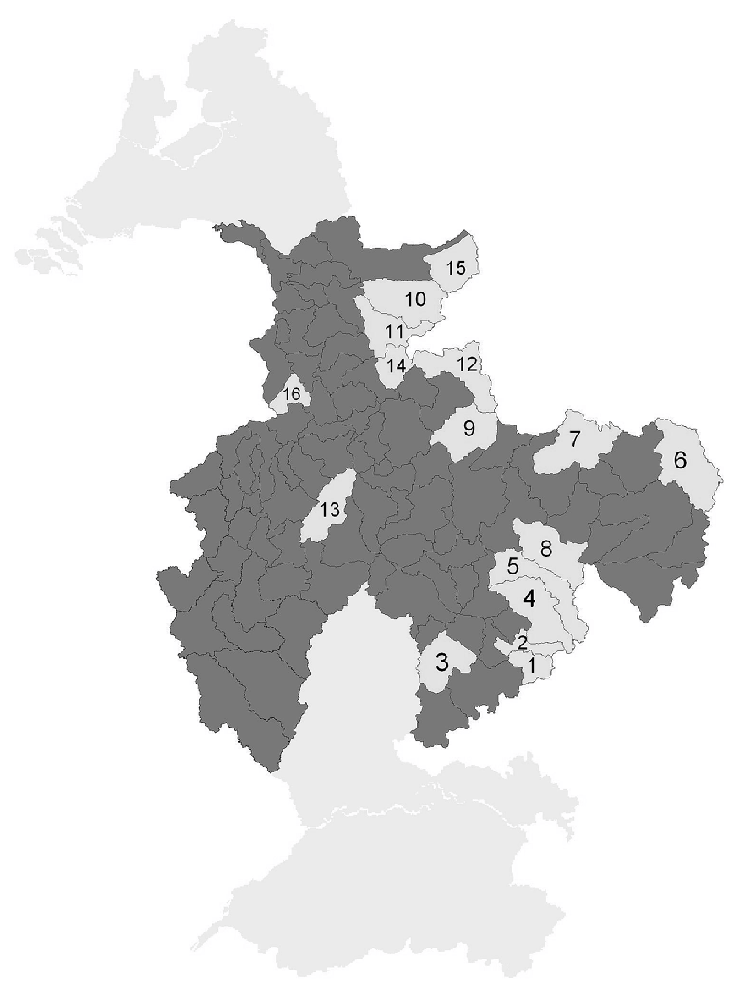

Fig. 5. Locations of the 16 selected catchments of the Rhine catchment.

of the catchments. Some of the catchment properties are summarized in Table 1 . Note that only headwater catchments were selected to avoid the uncertainties of inflow and routing.

A large number of different parameter vectors was considered for all of these catchments. The model's 5 conceptual parameters describing the runoff concentration using two reservoirs were selected for possible parameter vector transfer from one catchment to another. The other parameters, related to runoff formation in a spatially distributed manner, were estimated based on the soil land use and topographical information using transfer functions according to the regionalization procedure described in Hundecha and Bárdossy (2004). For each of the five selected model parameters, a range was fixed and a uniform distribution within the range was assumed to generate the candidate parameter vectors. No explicit dependence between the parameters was assumed. A large number of parameter vectors was generated according to these assumptions. Following this, the hydrological model was applied and the model performance was measured using an appropriate "quality" function. A typical performance measure is the Nash-Sutcliffe efficiency (NS):

$\mathrm{NS}=1-\frac{\sum_{t=1}^{T}\left(Q_{O}(t)-Q_{M}(t)\right)^{2}}{\sum_{t=1}^{T}\left(Q_{O}(t)-\overline{Q_{O}}\right)^{2}}$ where $Q_{O}(t)$ is the observed $Q_{M}(t)$ the modelle discharge at time $t . T$ is the time horizon and $\overline{Q_{O}}$ is the mean of the observed discharge. A daily time resulution is used for this purpose.

The model performance was calculated for each realisation of random parameter vectors. The set of good parameter vectors was defined as the subset of the generated vectors for which the NS exceeded $90 \%$ of the maximal NS value of the set for catchment $i$. This set, denoted as $D(i)$, was subsequently investigated in the parameter space. Due to the fact that 5 parameters were varied in this case is not possible to investigate the set of good parameters visually as in the case of the unit hydrograph. One or two dimensional projections might be misleading as demonstrated on Figs. 2 and 3. Therefore the characterization of the set $D(i)$ requires special methods.

In order to identify possible linear parameter dependencies, a principal component analysis (PCA)was carried out. This analysis showed that the effective linear dimension of $D(i)$ is dependent on the catchment and varies between 3 and 4, not 5. Subsequently, the Hausdorff dimension of the set $D(i)$ was estimated. The Hausdorff dimension offers a possibility to assign a (not necessarily integer) dimension to an arbitrary set of points. It measures the geometrical structure of the set (by identifying whether the are located on a line (not necessarily straight) or on a hypersurface. It is defined as the limit (Falconer, 1987)

$d_{H}(i)=\lim _{\varepsilon \rightarrow 0^{+}} \frac{\ln N(\varepsilon)}{\ln \varepsilon}$

with $N(\varepsilon)$ being the minimum number of open spheres of diameter $\varepsilon$ required to cover the set $D(i)$. Due to the high dimension of the parameter vector ( 5 parameters were considered) the available sample did not allow an accurate estimation of the Hausdorff dimension. However, a comparison of the $N(\varepsilon)$-s for a random set of the same cardinality and the set $D(i)$ indicated that the dimension of $D(i)$ is much lower than the linear dimension identified using PCA. The calculated values suggest that the Hausdorff dimension of the good parameter set is between 1.5 and 3 and depends on the catchment. A highly non-linear compensation of the model parameters is responsible for this low dimension. Note that for the example of the unit hydrograph (Nash cascade with two parameters), the Hausdorff dimension of the good parameter set slightly exceeds 1 (as the points are on one curve). Unfortunately there are no tools available to identify the analytical form of the lower dimensional manifold which contains the points of the set $D(i)$ for the HBV model. Thus a transformation of the parameters with a functional relationship remains very uncertain. Instead, all elements of the set $D(i)$ can be considered as candidates for the transfer of parameters from $i$ to another catchment $j$. They all have the property of representing a reasonable compensation of the individual parameters. Unfortunately not all of the elements of $D(i)$ deliver reasonable hydrographs for catchment $j$. 
Table 1. Properties of the 16 selected catchments.

\begin{tabular}{rllcccccc}
\hline Nr. & Location & River & Area $\left(\mathrm{km}^{2}\right)$ & Slope $(\%)$ & $1 /$ w ratio & Forest & Settlement & Agriculture \\
\hline 1 & Plochingen & Fils & 706 & 1.8358 & 2.2 & 0.376 & 0.162 & 0.460 \\
2 & Neustadt & Rems & 581 & 1.9769 & 6.0 & 0.388 & 0.164 & 0.448 \\
3 & Pforzheim & Enz & 1477 & 1.5265 & 1.08 & 0.605 & 0.128 & 0.267 \\
4 & Stein & Kocher & 1957 & 1.2475 & 2.84 & 0.378 & 0.113 & 0.508 \\
5 & Untergriesheim & Jagst & 1836 & 0.8710 & 7.89 & 0.262 & 0.103 & 0.634 \\
6 & Schwuerbitz & Main & 2426 & 1.5804 & 0.74 & 0.403 & 0.082 & 0.515 \\
7 & Wolfsmuenster & Fraenkische Saale & 2131 & 1.6342 & 1.87 & 0.372 & 0.080 & 0.548 \\
8 & Waldenhausen & Tauber & 1810 & 0.9983 & 2.62 & 0.217 & 0.092 & 0.689 \\
9 & Bad Viebel & Nidda & 1619 & 1.1501 & 1.43 & 0.301 & 0.139 & 0.560 \\
10 & Villigst & Ruhr & 2009 & 1.7733 & 2.21 & 0.620 & 0.121 & 0.247 \\
11 & Hagen-Hohenlimburg & Lenne & 1322 & 1.9611 & 2.39 & 0.758 & 0.143 & 0.091 \\
12 & Marburg & Lahn & 1666 & 1.5067 & 2.38 & 0.453 & 0.118 & 0.429 \\
13 & Martinstein & Nahe & 1435 & 1.5938 & 2.13 & 0.601 & 0.105 & 0.293 \\
14 & Betzdorf & Sieg & 755 & 1.9191 & 1.29 & 0.766 & 0.178 & 0.055 \\
15 & Lippstadt & Lippe & 1394 & 1.0127 & 1.25 & 0.339 & 0.127 & 0.530 \\
16 & Bliesheim & Erft & 604 & 1.0294 & 1.25 & 0.333 & 0.143 & 0.524 \\
\hline
\end{tabular}

In order to identify those elements of $D(i)$ which could be reasonably used for catchment $j$, a regionalisation of the discharge statistics was first carried out. For this purpose, the methodology described in Samaniego and Bárdossy (2005) was used. The mean annual discharges, $M_{Q}(j)$, and the standard deviation, $S_{Q}(j)$, of the daily discharges were estimated as a function of the catchment characteristics and statistics of the meteorological input; the estimator of the mean being of the form

$M_{Q}(j)=a_{0} \prod_{k=1}^{K} x_{k}(j)^{a_{k}}+\epsilon_{M}(j)$

and for the standard deviation

$S_{Q}(j)=b_{0} \prod_{k=1}^{K} x_{k}(j)^{b_{k}}+\epsilon_{S}(j)$

with $x_{k}(j)$ as the k-th characteristics of catchment $j$ and with $\epsilon_{M}(j)$ (or $\epsilon_{S}(j)$ ) being the corresponding estimation errors. The characteristics were selected in a stepwise manner from a large number of catchment descriptors listed in Samaniego and Bárdossy (2005). Parameters $a_{k}$ and $b_{k}$ are obtained after selection of the characteristics using a least squares approach. The descriptors consist of time dependent parameters (for example annual/seasonal precipitation, temperature) slowly changing parameters (for example land use) and time invariant parameters (for example soil and topography). Elements of $D(i)$ the good parameter set for catchment $i$, are taken to obtain a reasonable parameter set for catchment $j$. For each parameter vector $\theta$ the model is applied with the meteorological input of catchment $j$. The mean $\bar{Q}_{j}(\theta)$ and the standard deviation $s_{j}(\theta)$ are calculated from the simulated discharge series. These means and standard deviations are compared to regionalisation results for catchment $j$ obtained from Eqs. (4) and (5). For a given parameter vector $\theta$ if the mean and the standard deviation calculated from the modeled discharges are sufficiently close to the mean and the standard deviations obtained via regionalisation then the model parameter vector is judged to lead to a reasonable water balance and reasonable dynamics of the discharges on catchment $j$. Thus this parameter vector $\theta$ can be transferred from catchment $i$ to catchment $j$. Formally, if

$\left|M_{Q}(j)-\bar{Q}_{j}(\theta)\right|<k_{M} s_{M}(j)$

and

$\left|S_{Q}(j)-s_{j}(\theta)\right|<k_{S} s_{S}(j)$

then parameter vector $\theta$ is considered as a reasonable candidate for catchment $j ; s_{M}(j)$ and $s_{S}(j)$ being the standard deviations of the estimation errors $\epsilon_{M}(j)$ and $\epsilon_{S}(j)$ in Eqs. (4) and (5). The parameters $k_{M}$ and $k_{S}$ are selected such that a portion $\mathrm{p}$ (typically $90 \%$ ) of the estimated errors are below the limit. This way the number of transferable parameter sets from catchment $i$ to catchment $j$ becomes a sensible subset of $D(i)$. According to this procedure, only those parameter vectors that provide a reasonable water balance and daily variability of discharge for the target ungauged catchment are considered for transfer. For the case study the mean discharge was of secondary importance as the runoff formation parameters were not transferred directly from one catchment to the other. The reason for considering it is to avoid cases where water balances are obtained by filling up storages, and leading to non-stationary conditions. 


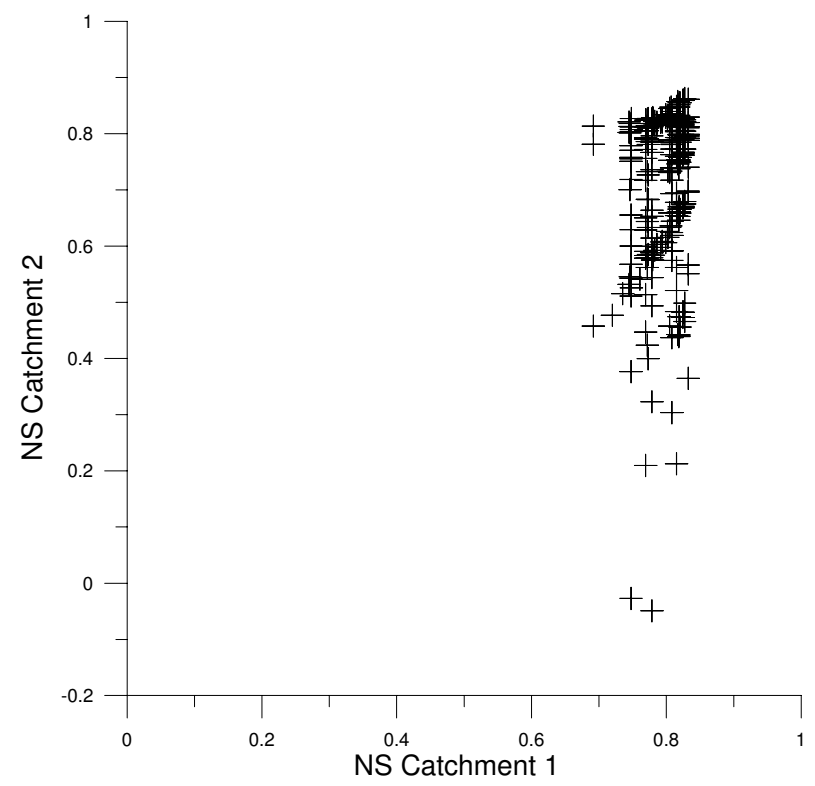

Fig. 6. Quality of parameter vectors for catchment 1 and catchment 11 for the transfer without considering the quality of the mean and the standard deviation.

\section{Summary and results}

A large set (2000 simulations) of possible candidates for 5 dimensional parameter vectors were generated at random. Uniform distributions were assumed for each parameter. The individual parameters were generated independently, without assuming any kind of parameter dependence. The HBV model was subsequently applied to all selected catchments using all possible candidate vectors. The model performance was expressed using the Nash-Sutcliffe coefficients. The regionalisation of the discharge statistics (annual mean flow and variance) was carried out using the approach described in Samaniego and Bárdossy (2005). To check the transferability of a parameter vector from catchment $i$ to $j$ the following steps were performed:

1. The Nash-Sutcliffe coefficient corresponding to the model for catchment $i$ was calculated for the selected parameter set $\theta$.

2. If the parameter set $\theta$ performed well on catchment $i$ (exceeding $90 \%$ of the performance of the best parameter vector) then it was added to the set $D(i)$ and applied for catchment $j$.

3. The statistics of the discharge series $\bar{Q}_{j}(\theta)$ and $s_{j}(\theta)$ corresponding to catchment $j$ were calculated using the HBV model.

4. If the annual discharge statistics did not differ much from the regionalisation (condition of Eqs. 6 and 7), the parameter vector was considered as a possible parameter vector for the modelling of catchment $j$.

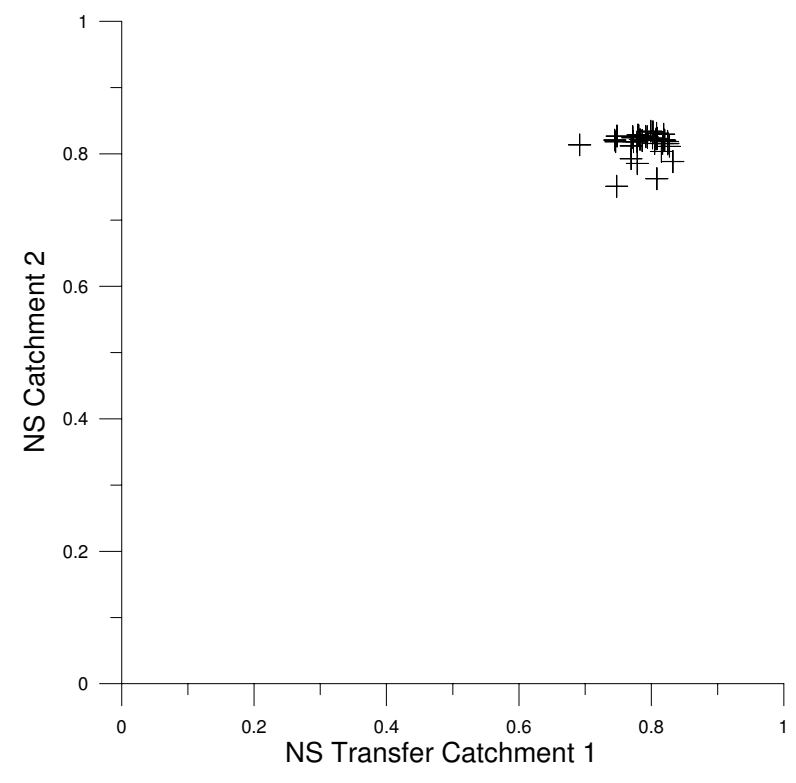

Fig. 7. Model performances for the transfer of parameter vectors from catchment 1 to catchment 11 for parameters fulfilling the conditions of the mean and the standard deviation.

5. In order to compare the results with observations for all retained candidate parameter vectors the model performance (NS value) on catchment $j$ was calculated.

To evaluate the performance of the method, the NS values of for catchment $i$ and $j$ were plotted as a scatter plot for all parameter vectors where their transfer was reasonable according to the annual statistics (step 4). Figure 6 shows an example for the transfer from catchment 1 to 11 , without considering the condition described in step 4 . Figure 7 shows the subset for the parameter vectors for which the mean and the standard deviation were estimated correctly by the criterion of step 4. As one can see in the second case, the selected parameter vectors yielded a good model performance on the catchment to which the parameter vector was transferred. The number of possible transferable candidate vectors was different depending on which pair of catchments was considered. The reason for the difference in number of transferable parameter vectors depends on the catchment characteristics. Figure 8 shows the observed and the modeled time series for catchment 11 using one of the set of parameters transferred from catchment 1 . As one can see, the performance of the transferred model is quantitatively very good.

Table 2 shows the best NS values obtained for each catchment and the mean NS values for transformed parameter sets from catchment $i$ to catchment $j$. One can see that if the criteria described in steps 2 and 4 of the above algorithm are fulfilled then the transfer leads to good performances. For two catchments (9 and 16) there were no good parameter sets among the generated sets. Catchments 3, 9, 15 and 16 are possible donors (their parameters can be used for others 
Table 2. Mean NS values for the parameter transfer from catchment to catchment.

\begin{tabular}{rcccccccccccccccc}
\hline Nr. & 1 & 2 & 3 & 4 & 5 & 6 & 7 & 8 & 9 & 10 & 11 & 12 & 13 & 14 & 15 & 16 \\
\hline 1 & $\mathrm{x}$ & 0.76 & - & 0.79 & 0.68 & 0.74 & 0.68 & 0.55 & - & 0.84 & 0.84 & 0.77 & 0.77 & 0.88 & - & - \\
2 & 0.83 & $\mathrm{x}$ & - & 0.80 & 0.69 & 0.74 & 0.68 & 0.55 & - & 0.84 & 0.85 & 0.77 & 0.78 & 0.89 & - & - \\
3 & - & 0.77 & $\mathrm{x}$ & 0.80 & 0.72 & 0.73 & 0.68 & 0.58 & - & 0.86 & 0.84 & 0.78 & - & 0.86 & - & - \\
4 & 0.82 & 0.75 & - & $\mathrm{x}$ & 0.68 & 0.74 & 0.68 & 0.54 & - & 0.84 & 0.84 & 0.76 & 0.77 & 0.88 & - & - \\
5 & 0.83 & 0.76 & - & 0.80 & $\mathrm{x}$ & 0.75 & 0.68 & 0.54 & - & 0.84 & 0.84 & 0.77 & 0.78 & 0.88 & - & - \\
6 & 0.81 & 0.72 & - & 0.78 & 0.67 & $\mathrm{x}$ & 0.68 & 0.54 & - & 0.84 & 0.83 & 0.76 & 0.76 & 0.88 & - & - \\
7 & 0.83 & 0.77 & - & 0.81 & 0.70 & 0.74 & $\mathrm{x}$ & 0.55 & - & 0.84 & 0.85 & 0.77 & 0.78 & 0.88 & - & - \\
8 & - & 0.76 & - & 0.79 & 0.73 & 0.75 & 0.68 & $\mathrm{x}$ & - & 0.86 & 0.85 & 0.77 & 0.77 & 0.88 & - & - \\
9 & - & - & - & - & 0.73 & - & - & 0.62 & $\mathrm{x}$ & 0.86 & - & 0.77 & - & - & - & - \\
10 & 0.84 & 0.78 & - & 0.81 & 0.71 & 0.74 & 0.68 & 0.55 & - & $\mathrm{x}$ & 0.85 & 0.77 & 0.78 & 0.88 & - & - \\
11 & 0.82 & 0.74 & - & 0.79 & 0.68 & 0.74 & 0.68 & 0.54 & - & 0.84 & $\mathrm{x}$ & 0.76 & 0.78 & 0.88 & - & - \\
12 & 0.81 & 0.76 & - & 0.80 & 0.68 & 0.74 & 0.67 & 0.54 & - & 0.84 & 0.84 & $\mathrm{x}$ & 0.78 & 0.88 & - & - \\
13 & 0.81 & 0.73 & - & 0.78 & 0.67 & 0.73 & 0.68 & 0.54 & - & 0.84 & 0.84 & 0.76 & $\mathrm{x}$ & 0.88 & - & - \\
14 & 0.80 & 0.72 & - & 0.77 & 0.67 & 0.73 & 0.68 & 0.54 & - & 0.84 & 0.83 & 0.76 & 0.76 & $\mathrm{x}$ & - & - \\
15 & 0.83 & 0.77 & - & 0.80 & 0.70 & 0.74 & 0.68 & 0.54 & - & - & 0.85 & 0.77 & 0.78 & 0.89 & $\mathrm{x}$ & - \\
16 & - & - & - & - & 0.73 & - & - & 0.62 & - & 0.86 & - & 0.77 & - & - & - & $\mathrm{x}$ \\
\hline Max & 0.86 & 0.82 & 0.74 & 0.82 & 0.73 & 0.76 & 0.68 & 0.62 & 0.14 & 0.86 & 0.87 & 0.80 & 0.80 & 0.90 & 0.74 & 0.46 \\
\hline
\end{tabular}

at least in a few cases) but bad a recipients. The remaining 10 catchments could be used both as donors and recipient of parameter sets. Note that the model performances of the transfered parameters are very close to the maximum performance, showing that the combination of the parameter selection criteria is reasonable. There number of transferable parameter sets varies from a very few $(\approx 1 \%$ of the good sets for catchment 1 could be used for 7 according to step 4 of the algorithm) to many ( $>90 \%$ for the pair 10 and 2) cases, indicating whether the manifolds of the good parameter sets are close or only intersect in a few cases. As for a few catchment the method did not lead to any transferable parameters this means that a regionalization for those catchments is not possible from the selected set. This fact limits the applicability of the method in a reasonable way - parameter vectors should not be transferred from a catchment to another if they behave very differently.

Both conditions the good performance on the donor catchment and the reasonable mean and standard deviation of the simulated discharges on the recipient catchment are important for the transfer of a parameter vector. Removing any of them leads to a strong deterioration of the results.

The reasons when and why transfers of parameters are possible cannot be derived simply from the catchment properties. Further research is needed to find an explanation for similar rainfall-runoff model behavior from catchment properties.

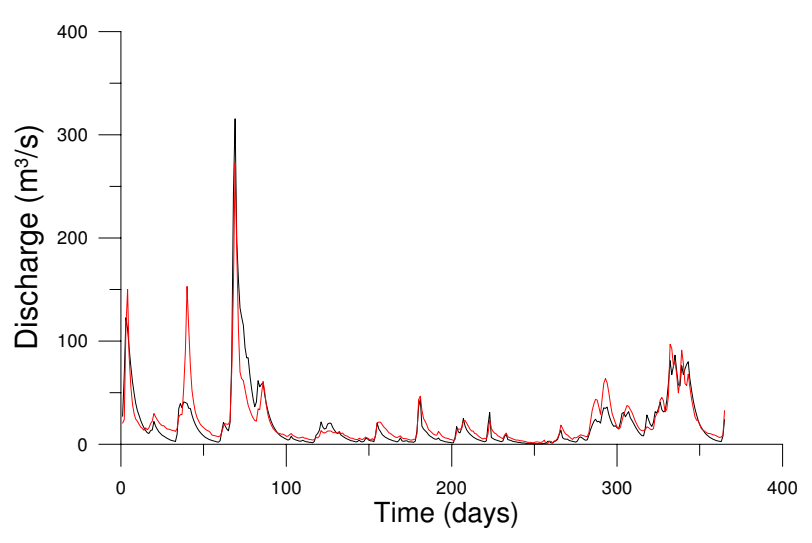

Fig. 8. Simulated (black) and observed discharges using model parameters transferred from catchment 1 (Plochingen, Fils) to catchment 11 (Hagen-Hohenlimburg, Lenne) for the year 1981, $\mathrm{NS}=0.84$.

\section{Conclusions}

Parameters of hydrological models cannot be identified as unique sets of values. This is mainly due to the fact that changes of one parameter can be compensated for by changes of one or more others, due to their interdependence. The non-linearity of the models implies that interpolation between parameter values can lead to unreasonable model parameters and results. Thus parameters should not be considered as individual values, but instead as parameter vectors "teams". Parameter vectors corresponding to a selected group of hydrological processes can be transferred from one catchment to another without any modification. This transfer 
is reasonable if the model obtained for the unobserved catchment gives good water balances and reproduces the variability of the daily discharges. The annual mean discharge and the variability can be regionalized using catchment characteristics and non linear regression models.

The presented approach is a kind of trial and error procedure. For some catchments it delivers good results, while for others, no parameter vectors can be found. In order to have a general methodology based on these ideas, further research on the dependence of parameters and catchment properties is required. The main lesson to be learned from this study is that regionalisation should not focus on relating individual parameter values to catchment properties but on relating them to compatible parameter sets, or vectors.

Further research is needed to understand why some catchments show a similar behavior by sharing good parameter sets for a given rainfall-runoff model. The question to what extent this depends on the model and to what extent on the catchment properties should also be investigated.

Acknowledgements. The author thanks the reviewers of this paper, B. Schaefli, A. Montanari and G. Pegram for their constructive comments which helped to improve the quality of this paper.

Edited by: E. Zehe

\section{References}

Abdulla, F. A. and Lettenmaier, D. P.: Development of regional estimation equations for a macroscale hydrologic model, J. Hydrol., 197, 230-257, 1997.

Ahmed, S. and de Marsily, G.: Comparison of geostatistical methods for estimating transmissivity using data transmissivity and specific capacity., Water Resour. Res., 23, 1717-1737, 1987.

Bergström, S.: The HBV model, in: Computer Models of Watershed Hydrology, Water Resour. Publications, Littleton, Colorado, USA, 1995.

Beven, K. and Freer, J.: Equifinality, data assimilation, and data uncertainty estimation in mechanistic modelling of complex environmental systems using the GLUE methodology, J. Hydrol., 249, 11-29, 2001.
Falconer, K.: The Geometry of Fractal Sets, Cambridge University Press, 1987.

Gupta, H., Sorooshian, S., and Yapo, P.: Toward improved calibration of hydrologic models: Multiple and noncommensurable measures of information, Water Resour. Res., 34(4), 751-763, 1998.

Heuvelmans, G., Muys, B., and Feyen, J.: Regionalisation of the parameters of a hydrological model: Comparison of linear regression models with artificial neural nets., J. Hydrol., 319, 245-265, 2006.

Hundecha, Y. and Bárdossy, A.: Modeling of the effect of land use changes on the runoff generation of a river basin through parameter regionalization of a watershed model, J. Hydrol., 292, 281-295, 2004.

Kokkonen, T., Jakeman, A., Young, P., and Koivusalo, H.: Predicting daily flows in ungauged catchments: model regionalization from catchment descriptors at the Coweeta Hydrologic Laboratory, North Carolina., Hydrol. Processes, 11, 2219-2238, 2003.

Kuczera, G. and Mroczkowski, M.: Assessment of hydrologic parameter uncertainty and the worth of data, Water Resour. Res. 34(6), 1481-1489, 1998.

Madsen, H.: Parameter estimation in distributed hydrological catchment modeling using automatic calibration with multiple objectives, Adv. Water Resour., 26, 205-216, 2003.

McIntyre, N., Lee, H., Wheater, H., Young, A., and Wagener, T. Ensemble predictions of runoff in ungauged catchments., Water Resour. Res., 41(12), W12434, doi:10.1029/2005WR004289, 2005.

Nash, J.: A unit hydrograph study with particular reference to British catchments, Proc. Inst. Civil. Eng., 17, 249-282, 1960.

Nash, J. and Sutcliffe, J.: River flow forecasting through conceptual models. 1. A discussion of principles, J. Hydrol., 10, 282-290, 1970.

Parajka, J., Merz, R., and Bloschl, G.: A comparison of regionalisation methods for catchment model parameters., Hydrol. Earth Syst. Sci., 9, 157-171, 2005, http://www.hydrol-earth-syst-sci.net/9/157/2005/.

Samaniego, L. and Bárdossy, A.: Robust parametric models of runoff characteristics at the mesoscale, J. Hydrol., 303, 136-151, 2005. 